\title{
Low-Loss Polymer-Based Ring Resonator for Resonant Integrated Optical Gyroscopes
}

\author{
Guang Qian, ${ }^{1,2,3}$ Jie Tang, ${ }^{1,2,3}$ Xiao-Yang Zhang, ${ }^{2,3,4}$ Ruo-Zhou Li, ${ }^{2,3,4}$ \\ Yu Lu, ${ }^{2,3,4}$ and Tong Zhang ${ }^{1,2,3,4}$ \\ ${ }^{1}$ School of Instrument Science and Engineering, Southeast University, Nanjing 210096, China \\ ${ }^{2}$ Key Laboratory of Micro-Inertial Instrument and Advanced Navigation Technology, Ministry of Education, Nanjing 210096, China \\ ${ }^{3}$ Suzhou Key Laboratory of Metal Nano-Optoelectronic Technology, Suzhou Research Institute of Southeast University, \\ Suzhou 215123, China \\ ${ }^{4}$ School of Electronic Science and Engineering, Southeast University, Nanjing 210096, China
}

Correspondence should be addressed to Tong Zhang; tzhang@seu.edu.cn

Received 28 February 2014; Revised 25 April 2014; Accepted 28 April 2014; Published 22 May 2014

Academic Editor: Qin Chen

Copyright (C) 2014 Guang Qian et al. This is an open access article distributed under the Creative Commons Attribution License, which permits unrestricted use, distribution, and reproduction in any medium, provided the original work is properly cited.

Waveguide ring resonator is the sensing element of resonant integrated optical gyroscope (RIOG). This paper reports a polymerbased ring resonator with a low propagation loss of about $0.476 \mathrm{~dB} / \mathrm{cm}$ for RIOG. The geometrical parameters of the waveguide and the coupler of the resonator were optimally designed. We also discussed the optical properties and gyroscope performance of the polymer resonator which shows a high quality factor of about $10^{5}$. The polymer-based RIOG exhibits a limited sensitivity of less than $20 \mathrm{deg} / \mathrm{h}$ for the low and medium resolution navigation systems.

\section{Introduction}

Resonant integrated optical gyroscope (RIOG) is a high performance rotation sensor based on the Sagnac effect, in which an optical waveguide ring resonator is utilized as the sensing element [1-3]. Due to the excellent advantages of compactness, stability, reliability, and ease of fabrication, RIOGs have attracted much attention in the field of inertial navigation [4-6]. Meanwhile, the less complex and cheaper gyroscopes suited for mass production are urgently required, especially in the low and medium resolution fields such as car navigation and robotics.

Optical polymeric materials permit the mass production of low-cost integrated optical devices and circuits on a planar substrate $[7,8]$. Polymer-based optical waveguide is the basic light transmission medium for integrated optical device. The polymer-based integrated optical devices have been widely used in many laboratories worldwide and some achieved commercialization $[9,10]$. Especially, the polymer-based optical ring resonators have become the focus of research due to their good optical characteristics [11] and have been widely used in integrated optical devices such as modulators [1214], filters [15, 16], sensors [17], and optical communication systems [18]. However, these applied polymer ring resonators are not suitable for the sensing elements of RIOGs due to the characteristics of small diameter and high propagation loss. The polymer-based resonator used in RIOG requires a much larger diameter and a lower propagation loss because the performance of gyroscope mainly depends on the enclosed area and the propagation loss of the waveguide resonator $[3,19]$.

In this paper, we reported a polymer-based resonator with a big diameter and a low propagation loss for constructing the polymer-based RIOG. We optimally designed the coupler and analyzed the optical properties and corresponding RIOG performance of the polymer-based resonator.

\section{Materials and Methods}

Figure 1(a) shows the scheme of the polymer ring resonator for RIOG. It consists of a bus waveguide and a ring waveguide. For RIOG, the ring resonator is the rotation sensing element 


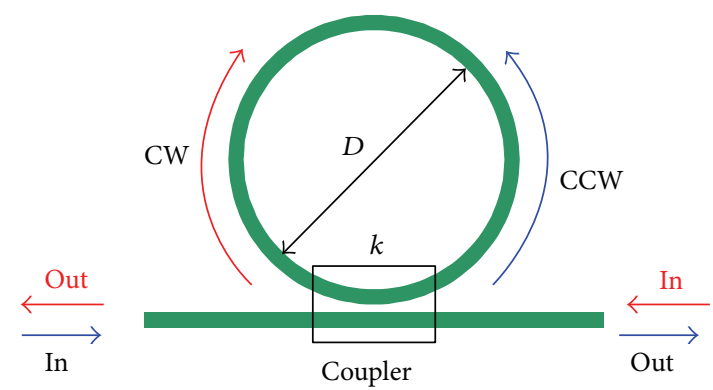

(a)

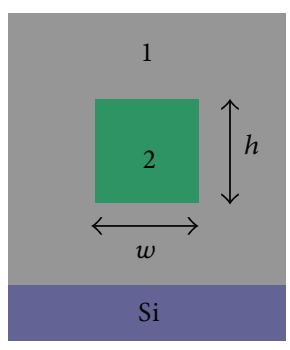

(b)

FIGURE 1: (a) Configuration of the polymer-based ring resonator for RIOG. (b) Cross section view of the geometry of the polymer waveguide.

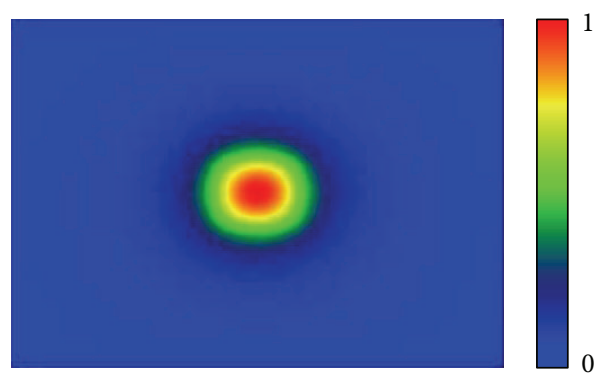

(a)

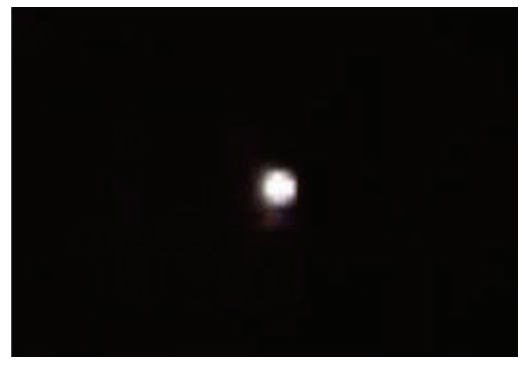

(b)

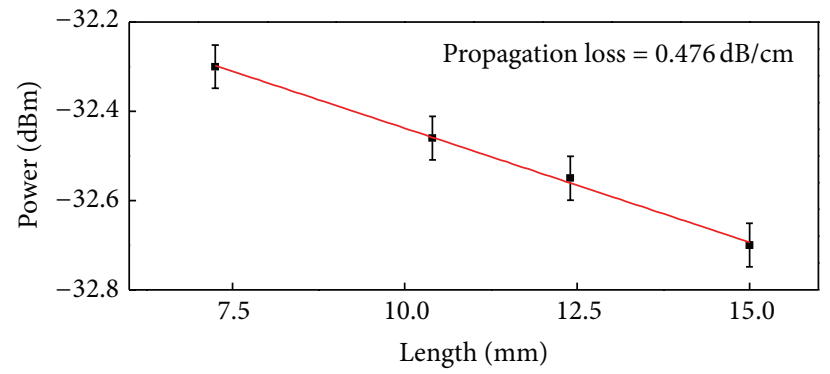

(c)

FIGURE 2: Simulation result (a) and measurement photograph (b) of optical field distribution of the polymer waveguide with parameters $w=5 \mu \mathrm{m}$ and $h=5 \mu \mathrm{m}$. (c) Propagation loss measurement of the polymer waveguide.

in which two beams with equal power launch into the two input ports of the bus waveguide, respectively. The input light is split into two beams by the coupler. One beam couples into the ring resonator with coupling ratio $k$, while the other beam transmits through the coupler directly. For polymer-based RIOG, a resonant frequency difference between the clockwise (CW) beam and the counterclockwise (CCW) beam in the ring resonator shown in Figure 1(a) will arise correspondingly when there is a rotation. The resonant frequency difference is proportional to the rotation ratio.

Figure 1(b) shows the configuration of the polymer waveguide from the cross section. A polymer stripe with a width of $w$ and a thickness of $h$ is buried in polymer 1 . The refractive index of polymer 1 and polymer 2 is 1.45 and 1.46, respectively. The refractive index contrast of the cladding and the buried core is 0.01 . The parameters were designed aiming at having an optimal coupling ratio $k$ and a low propagation loss.

\section{Results and Discussion}

In this section, we researched the light propagation in the polymer waveguide, the influence of the gap between the bus and the ring waveguide on coupling ratio, and the optical properties of the polymer ring resonator and its corresponding gyroscope performance.

3.1. Coupler Design. For the view of coupler in the ring resonator shown in Figure 1(a), the buried core of the waveguide needs a small width and a small thickness [20]. The obtained optimal thickness $(h)$ and width $(w)$ of the polymer waveguide shown in Figure 1(b) are all equal to $5 \mu \mathrm{m}$. Figure 2(a) shows the simulation result of optical field distribution of the utilized polymer waveguide at an incident wavelength of $1550 \mathrm{~nm}$. The calculated effective refractive index of the polymer waveguide is 1.454. Figure 2(b) shows the measurement result of optical field distribution in the 


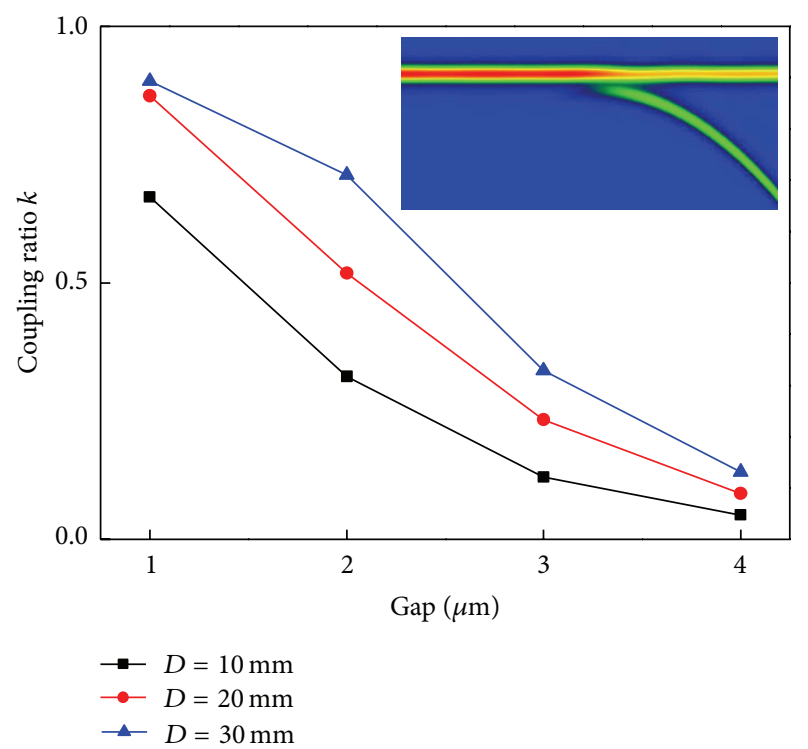

FIGURE 3: Influences of the minimum gap and the resonator diameter on coupling ratio $k$. The inset shows the power transmission in the bus and ring polymer waveguides with $D=10 \mathrm{~mm}$ and gap = $2 \mu \mathrm{m}$.

polymer waveguide. Figure 2(c) shows the propagation loss measurement of the polymer waveguide by the traditional cut-back method. The measured propagation loss is about $0.476 \mathrm{~dB} / \mathrm{cm}$ at an incident wavelength of $1550 \mathrm{~nm}$. The results show that this polymer waveguide has a good performance for light propagation.

To construct a polymer-based ring resonator, the relationship between the minimum gap of bus/ring waveguide and the coupling ratio $k$ of the coupler is studied. Coupling ratio $k$ is the significant parameter of the coupler which represents the fraction of power coupled into the ring resonator from the input port of the bus waveguide [21]. The main factor of coupling ratio $k$ is the geometrical dimension of the minimum gap between the bus and ring waveguide. Figure 3 shows the influences of the minimum gap and the diameter of resonator on the coupling ratio $k$ with parameters $w=5 \mu \mathrm{m}$ and $h=5 \mu \mathrm{m}$ at an incident wavelength of $1550 \mathrm{~nm}$.

From the calculated results, we can see that the coupling ratio $k$ is highly dependent on the diameter $D$ and the minimum gap of the bus/ring waveguide. With an increasing minimum gap, the value of coupling ratio $k$ decreases because the bigger gap reduces the power coupling between the bus and ring waveguide. In addition, the coupling ratio $k$ can be improved by increasing the diameter of the polymer resonator, which is mainly because of the increase of the coupling length between the bus and ring waveguide. The inset of Figure 3 shows the power transmission in the bus and ring polymer waveguides with parameters $D=10 \mathrm{~mm}$ and gap $=2 \mu \mathrm{m}$ at wavelength of $1550 \mathrm{~nm}$. The corresponding calculated result of coupling ratio $k$ is about 0.32 shown in Figure 3.

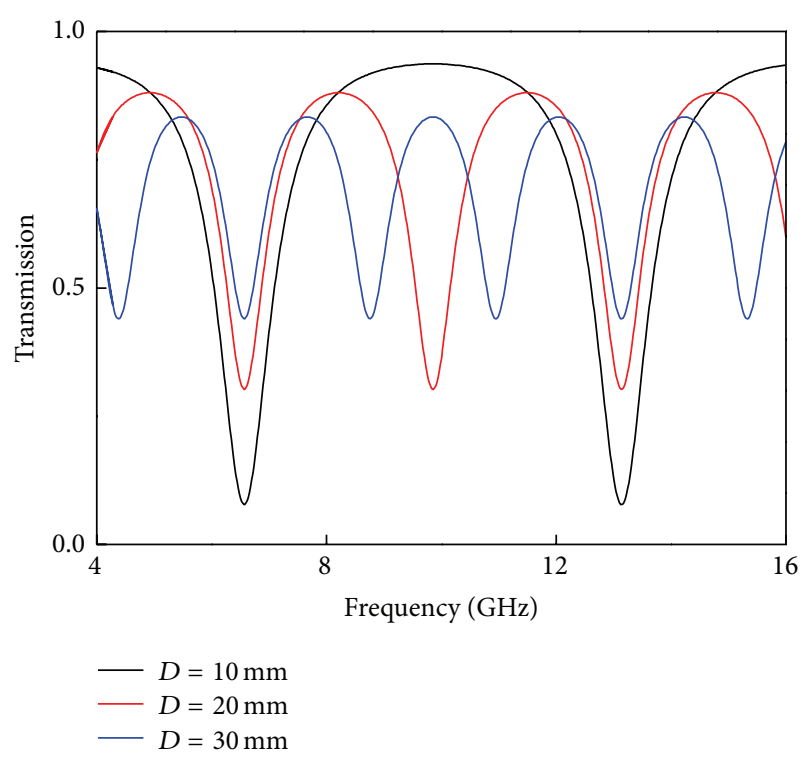

FIGURE 4: Transmission spectra of the polymer ring resonator with different diameter of $10 \mathrm{~mm}, 20 \mathrm{~mm}$, and $30 \mathrm{~mm}$ at $k=0.32$.

3.2. Optical Properties of the Polymer Resonator. For the polymer-based ring resonator, the propagation loss, coupling ratio, and circumference of the resonator are crucial parameters to determine its performance. The transmission function of a ring resonator is given by $[6,22]$

$$
T=\left|\frac{E_{\text {out }}}{E_{\text {in }}}\right|^{2}=\frac{(1-k)+\gamma^{2}-2 \gamma \sqrt{1-k} \cos (\beta L)}{1+\gamma^{2}(1-k)-2 \gamma \sqrt{1-k} \cos (\beta L)},
$$

where $\gamma=\exp (-\alpha L), k$ is the coupling ratio, $\beta$ is the optical propagation constant, and $L$ is the circumference of the polymer resonator. $\alpha$ is the propagation loss of the polymer waveguide which mainly contains material loss, bending loss, and scatter loss. The material loss is power loss absorbed inside the material due to the absorption and the imperfections in the bulk waveguide material [20]. Bending loss is power leakage that occurred in the curved part of waveguide and primarily determined by the bend radius [23]. Scatter loss is caused by physical surface roughness especially on the side walls of waveguide produced typically during its fabrication [24].

Equation (1) indicates that the propagation loss of the polymer waveguide significantly influences the transmission property of the ring resonator. The calculated bending loss of the proposed polymer resonator with bend radius $5 \mathrm{~mm}$ is about $0.01 \mathrm{~dB} / \mathrm{cm}$. This value is so small that it can be neglected. The measured propagation loss of the polymer waveguide is $0.476 \mathrm{~dB} / \mathrm{cm}$. Figure 4 shows the transmission spectra of the polymer ring resonator with different diameters at $k=0.32$. When the propagation loss $\alpha$ and the coupling ratio $k$ are fixed, the transmission spectrum is determined by the circumference of the polymer ring resonator. From Figure 4, we can see that the free spectral range (FSR) and the resonant depth of the ring resonator all decrease with the increase of the diameter of ring resonator. For a given material 
TABLE 1: Performance parameters of the polymer resonator with $D=10 \mathrm{~mm}$ and gap $=2 \mu \mathrm{m}$.

\begin{tabular}{lcccccc}
\hline Parameters & Loss $(\mathrm{dB} / \mathrm{cm})$ & Coupling ratio & FSR $(\mathrm{GHz})$ & FWHM $(\mathrm{GHz})$ & $Q\left(10^{5}\right)$ & Finesse \\
\hline Value & 0.476 & 0.32 & 6.56 & 1.07 & 1.8 & 6.13 \\
\hline
\end{tabular}

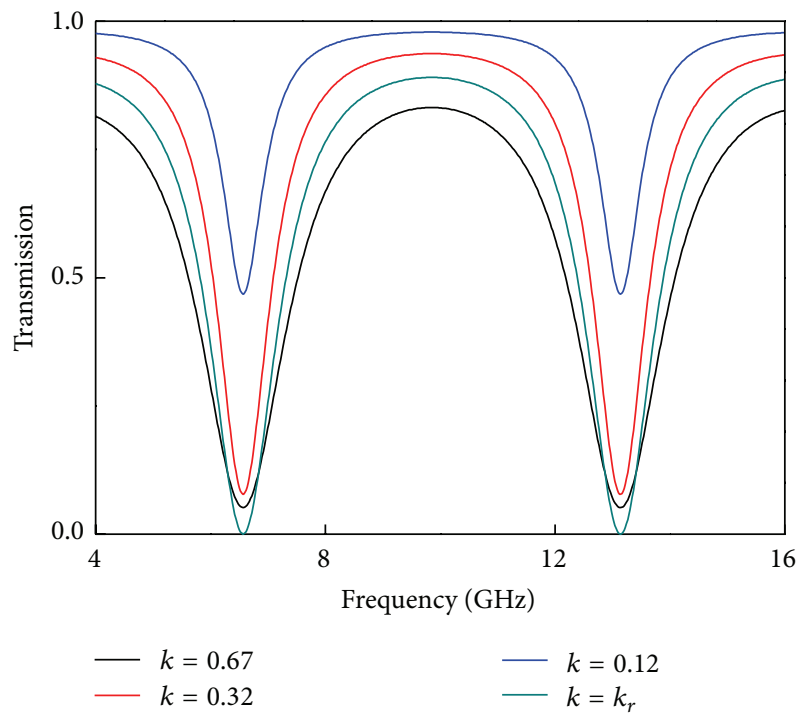

FIGURE 5: Transmission spectra of the polymer ring resonator with $D=10 \mathrm{~mm}$ and coupling ratio $k$ of $0.67,0.32,0.12$, and $k_{r}$.

(i.e., a fixed loss factor), the output signal decreases dramatically with an increasing diameter $D$ shown in Figure 4. This is mainly because the long propagation length of the large diameter resonator results in large power attenuation.

For RIOG, a large resonant depth is beneficial for rotation signal detection. The resonant depth of the ring resonator is also related to the coupling ratio $k$. Figure 5 shows the influence of coupling ratio $k$ on the resonance of polymer ring resonator.

The minimum gap dimension of the bus/ring waveguide corresponding to the coupling ration $k$ of $0.67,0.32$, and 0.12 is $1 \mu \mathrm{m}, 2 \mu \mathrm{m}$, and $3 \mu \mathrm{m}$, respectively. The resonant depth reaches the largest value at the critical coupling point $(k=$ $k_{r}=1-\gamma^{2}$ ). Figure 5 indicates that the coupling ratio $k$ of the ring resonator influences not only the resonant depth, but also the full width at the half maximum of the frequency (FWHM). The three ring resonators have the same free spectral range because of the same diameter.

For polymer ring resonator with $D=10 \mathrm{~mm}$ and gap = $2 \mu \mathrm{m}$, the free spectral range (FSR), FWHM, and quality factor $(Q)$ are $6.56 \mathrm{GHz}, 1.07 \mathrm{GHz}$, and $1.8 \times 10^{5}$, respectively. The detailed performance parameters are shown in Table 1.

3.3. Gyroscope Performance. The sensitivity of a resonant integrated optical gyroscope is typically limited by the shot noise at the two photodetectors included in the readout system. It can be estimated by the following formula [25]:

$$
\begin{gathered}
\delta \Omega \cong \frac{\lambda L}{4 A} \cdot \frac{\sqrt{2} \cdot \delta f_{1 / 2}}{\mathrm{SNR}}=\frac{\lambda}{D} \cdot \frac{\sqrt{2} \cdot \delta f_{1 / 2}}{\mathrm{SNR}}, \\
\delta f_{1 / 2}=\frac{2 c}{\pi n L} \sin ^{-1} \frac{1-\gamma \sqrt{1-k}}{\sqrt{2\left[1+(1-k) \gamma^{2}\right]}}, \\
\mathrm{SNR}=\sqrt{\frac{\eta t P_{\text {in }}}{2 h f}} \frac{T_{\max }-T_{\min }}{\sqrt{T_{\max }}},
\end{gathered}
$$

where $D$ is the diameter of the polymer-based ring resonator, $\lambda$ is the sensor operating wavelength, and $\delta f_{1 / 2}$ is the FWHM. $T_{\max }$ is maximum value of $T$ when $\cos (\beta L)=-1 . T_{\min }$ is the minimum value of $T$ when $\cos (\beta L)=1$. SNR is the signal to noise ratio of the detective system which is related to quantum efficiency $\eta$, integral time of photodetector $t$, Planck constant $h$, frequency of light $f$, and input power of light $P_{\text {in }}$.

Equation (2)-(4) shows that the value of limited sensitivity $\delta \Omega$ is negatively related to the closed area of the ring resonator. The results indicate that a large closed area can lead to an improvement of sensitivity of resonant optical gyroscope. The limited sensitivity can be also improved by reducing the $\delta f_{1 / 2}$ which is determined by the propagation loss $\alpha$, the circumference of the polymer resonator $L$, and the coupling ratio $k$. In addition, (2) indicates that the gyroscope's sensitivity can be also improved by increasing the signal to noise ratio of the detective system. The input power of light is proportional to the signal to noise ratio of the detective system. Therefore, improvement of the input power of light is an effective way to achieve a higher sensitivity when the structure of resonant optical gyroscope is fixed.

Figure 6 shows the relationships between the coupler ratio $k$ and the sensitivity of the polymer-based gyroscope at different diameter $D$ of ring resonator. It can be observed that the sensitivity of polymer-based resonant integrated optical gyroscope increases with the increase of diameter $D$ of the polymer resonator when coupling ratio $k$ is a constant. Furthermore, there is an optimal value of coupling ratio $k$ for the limited sensitivity of gyroscope. The optimal limited sensitivity of the polymer-based gyroscope with $D=$ $10 \mathrm{~mm}$ resonator is less than $20 \mathrm{deg} / \mathrm{h}$. For RIOG with $D=$ $30 \mathrm{~mm}$, the optimal limited sensitivity is about $5 \mathrm{deg} / \mathrm{h}$. The gyroscope with this sensitivity can be widely used in the inertial navigation field.

\section{Conclusions}

A polymer-based ring resonator is proposed for constructing resonant integrated optical gyroscope. We optimally designed 


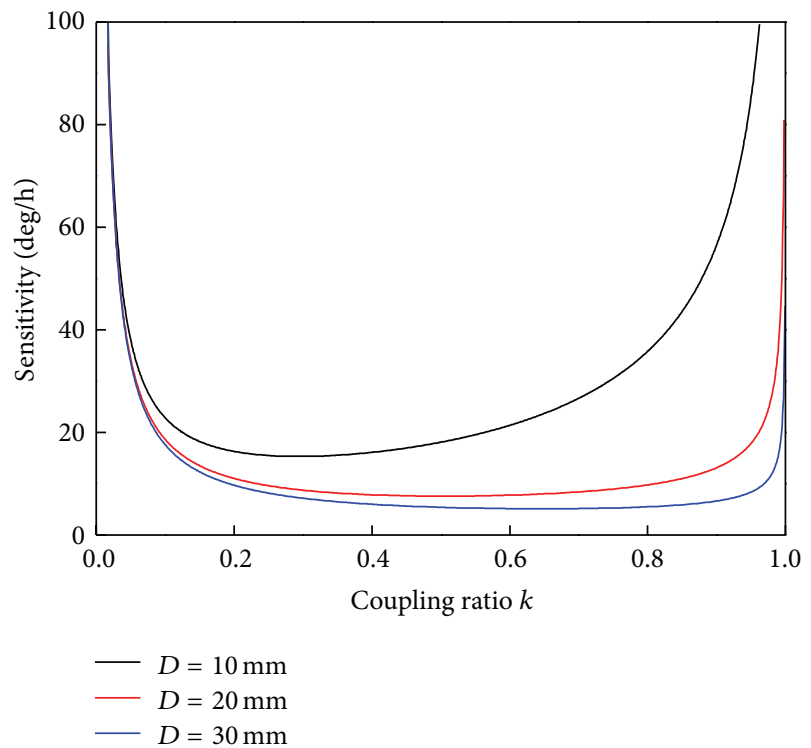

FIGURE 6: The sensitivity of polymer-based RIOG versus the coupling ratio for various resonator diameters.

and fabricated the polymer waveguide with good measurement results. The measured propagation loss of the polymer waveguide at $1550 \mathrm{~nm}$ is about $0.476 \mathrm{~dB} / \mathrm{cm}$. The analysis about coupler shows that the value of coupling ratio is mainly determined by the gap of the bus/ring waveguide and the diameter of ring resonator. The optical properties and gyroscope performance of the polymer ring resonator are discussed which shows a potential shot noise limited sensitivity less than $20 \mathrm{deg} / \mathrm{h}$. The polymer-based integrated optical gyroscopes permitted mass production will be a promising candidate of the new generation gyroscopes on a chip.

\section{Conflict of Interests}

The authors declare that there is no conflict of interests regarding the publication of this paper.

\section{Acknowledgments}

This work is supported by NSFC under Grant no. 61307066, Doctoral Fund of Ministry of Education of China under Grant nos. 20110092110016 and 20130092120024, Natural Science Foundation of Jiangsu Province under Grant no. BK20130630, the National Basic Research Program of China (973 Program) under Grant no. 2011CB302004, and the Foundation of Key Laboratory of Micro-Inertial Instrument and Advanced Navigation Technology, Ministry of Education, China, under Grant no. 201204.

\section{References}

[1] K. Suzuki, K. Takiguchi, and K. Hotate, "Monolithically integrated resonator microoptic gyro on silica planar lightwave circuit," Journal of Lightwave Technology, vol. 18, no. 1, pp. 66-72, 2000.

[2] T. Zhang, G. Qian, Y. Y. Wang et al., "Integrated optical gyroscope using active Long-range surface plasmon-polariton waveguide resonator," Scientific Reports, vol. 4, article 3855, 2014.

[3] X.-Y. Zhang, C.-L. Ji, T. Zhang, and Y.-P. Cui, "Analysis of ring resonator of integrated optical waveguide gyroscope," in 3rd International Symposium on Advanced Optical Manufacturing and Testing Technologies: Advanced Optical Manufacturing Technologies, vol. 6722 of Proceedings of SPIE, p. 94, July 2007.

[4] C. Ciminelli, F. Dell'Olio, C. E. Campanella, and M. N. Armenise, "Photonic technologies for angular velocity sensing," Advances in Optics and Photonics, vol. 2, no. 3, pp. 370-404, 2010.

[5] M. Á. Guillén-Torres, E. Cretu, N. A. F. Jaeger, and L. Chrostowski, "Ring resonator optical gyroscopes-parameter optimization and robustness analysis," Journal of Lightwave Technology, vol. 30, no. 12, Article ID 6157591, pp. 1802-1817, 2012.

[6] F. Zhang and J. W. Lit, "Direct-coupling single-mode fiber ring resonator," Journal of the Optical Society of America A, vol. 5, no. 8, pp. 1347-1355, 1988.

[7] L. Eldada and L. W. Shacklette, "Advances in polymer integrated optics," IEEE Journal on Selected Topics in Quantum Electronics, vol. 6 , no. 1, pp. 54-68, 2000.

[8] J.-G. Chen, T. Zhang, J.-S. Zhu et al., "Low-loss planar optical waveguides fabricated from polycarbonate," Polymer Engineering and Science, vol. 49, no. 10, pp. 2015-2019, 2009.

[9] H. Ma, A. K. Jen, and L. R. Dalton, "Polymer-based optical waveguides: materials, processing, and devices," Advanced Materials, vol. 14, no. 19, pp. 1339-1365, 2002.

[10] D.-M. Zhang, C. Chen, X. Sun et al., "Optical properties of $\operatorname{Er}(\mathrm{DBM}) 3 \mathrm{Phen}$-doped polymer and fabrication of ridge waveguide," Optics Communications, vol. 278, no. 1, pp. 90-93, 2007.

[11] X.-Y. Zhang, T. Zhang, and J.-G. Chen, "Thermooptical polymer ring resonator integrated with tunable directional coupler," in 2008 International Conference on Optical Instruments and Technology: Microelectronic and Optoelectronic Devices and Integration, vol. 7158 of Proceedings of SPIE, p. 71580X, November 2008.

[12] B. Bortnik, Y. Hung, H. Tazawa et al., "Electrooptic polymer ring resonator modulation up to $165 \mathrm{GHz}$," IEEE Journal on Selected Topics in Quantum Electronics, vol. 13, no. 1, pp. 104$110,2007$.

[13] X.-Y. Zhang, T. Zhang, X.-J. Xue et al., “Tunable optical ring resonator integrated with asymmetric machzehnder interferometer," Journal of Lightwave Technology, vol. 28, no. 17, pp. 2512-2520, 2010.

[14] M. Gould, T. Baehr-Jones, R. Ding et al., "Silicon-polymer hybrid slot waveguide ring-resonator modulator," Optics Express, vol. 19, no. 5, pp. 3952-3961, 2011.

[15] S. Yamagata, Y. Yanagase, and Y. Kokubun, "Wide-range tunable microring resonator filter by thermo-optic effect in polymer waveguide," Japanese Journal of Applied Physics 1: Regular Papers and Short Notes and Review Papers, vol. 43, no. 8, supplement, pp. 5766-5770, 2004.

[16] P. Rabiei, W. H. Steier, C. Zhang, and L. R. Dalton, "Polymer micro-ring filters and modulators," Journal of Lightwave Technology, vol. 20, no. 11, pp. 1968-1975, 2002. 
[17] J. Ren, L.-H. Wang, X.-Y. Han et al., "Organic silicone sol-gel polymer as a noncovalent carrier of receptor proteins for labelfree optical biosensor application," ACS Applied Materials \& Interfaces, vol. 5, no. 2, pp. 386-394, 2012.

[18] A. Chen, H. Sun, A. Pyayt, L. R. Dalton, J. Luo, and A. K.Y. Jen, "Microring resonators made in poled and unpoled chromophore-containing polymers for optical communication and sensors," IEEE Journal on Selected Topics in Quantum Electronics, vol. 14, no. 5, pp. 1281-1288, 2008.

[19] D. M. Shupe, "Fiber resonator gyroscope: sensitivity and thermal nonreciprocity," Applied Optics, vol. 20, no. 2, pp. 286-289, 1981.

[20] B. Seo, S. Kim, H. Fetterman, W. Steier, D. Jin, and R. Dinu, "Design of ring resonators using electro-optic polymer waveguides," Journal of Physical Chemistry C, vol. 112, no. 21, pp. 79537958, 2008.

[21] H. Hsiao and K. A. Winick, "Planar glass waveguide ring resonators with gain," Optics Express, vol. 15, no. 26, pp. 1778317797, 2007.

[22] A. Yariv, "Universal relations for coupling of optical power between microresonators and dielectric waveguides," Electronics Letters, vol. 36, no. 4, pp. 321-322, 2000.

[23] Y. A. Vlasov and S. J. McNab, "Losses in single-mode siliconon-insulator strip waveguides and bends," Optics Express, vol. 12, no. 8, pp. 1622-1631, 2004.

[24] C. Chao and L. J. Guo, "Reduction of surface scattering loss in polymer microrings using thermal-reflow technique," IEEE Photonics Technology Letters, vol. 16, no. 6, pp. 1498-1500, 2004.

[25] W. Li, T. Zhang, X.-Y. Zhang, S.-Q. Zhu, and D.-X. Yang, "Theoretical analysis of long-range surface plasmon-polariton waveguide gyroscope," Nanoscience and Nanotechnology Letters, vol. 5, no. 2, pp. 126-129, 2013. 

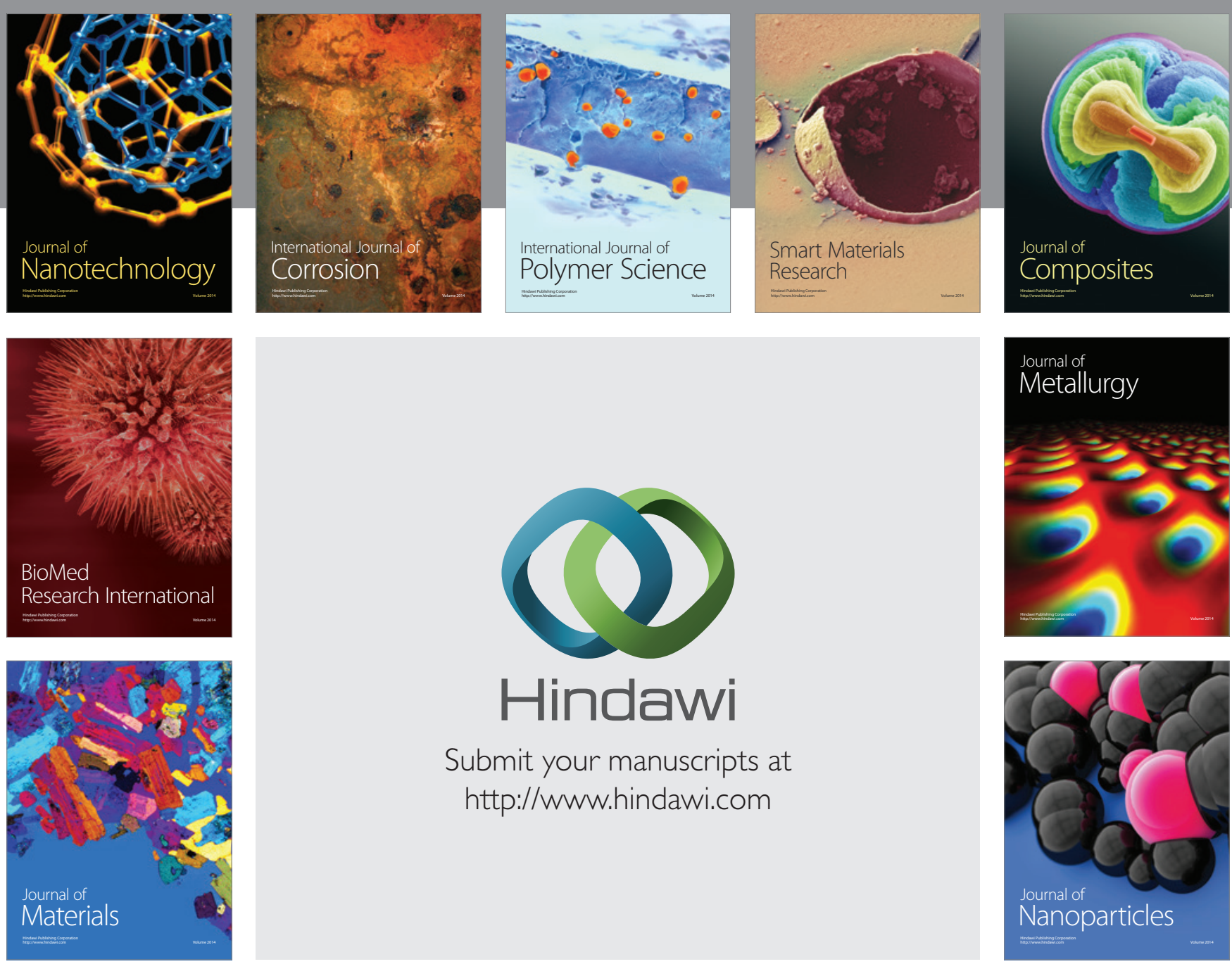

Submit your manuscripts at http://www.hindawi.com
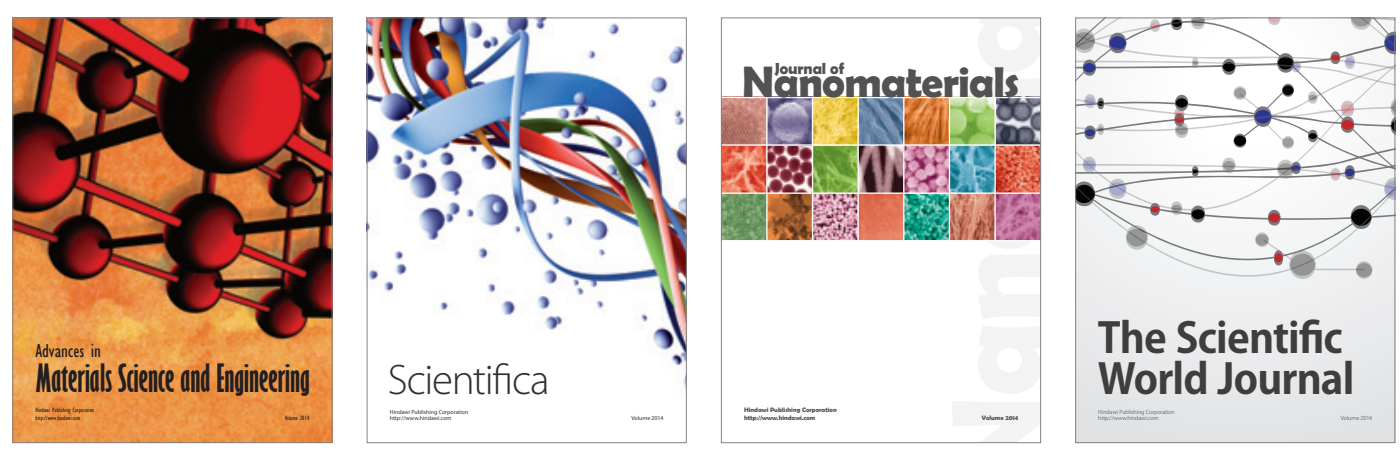

\section{The Scientific World Journal}
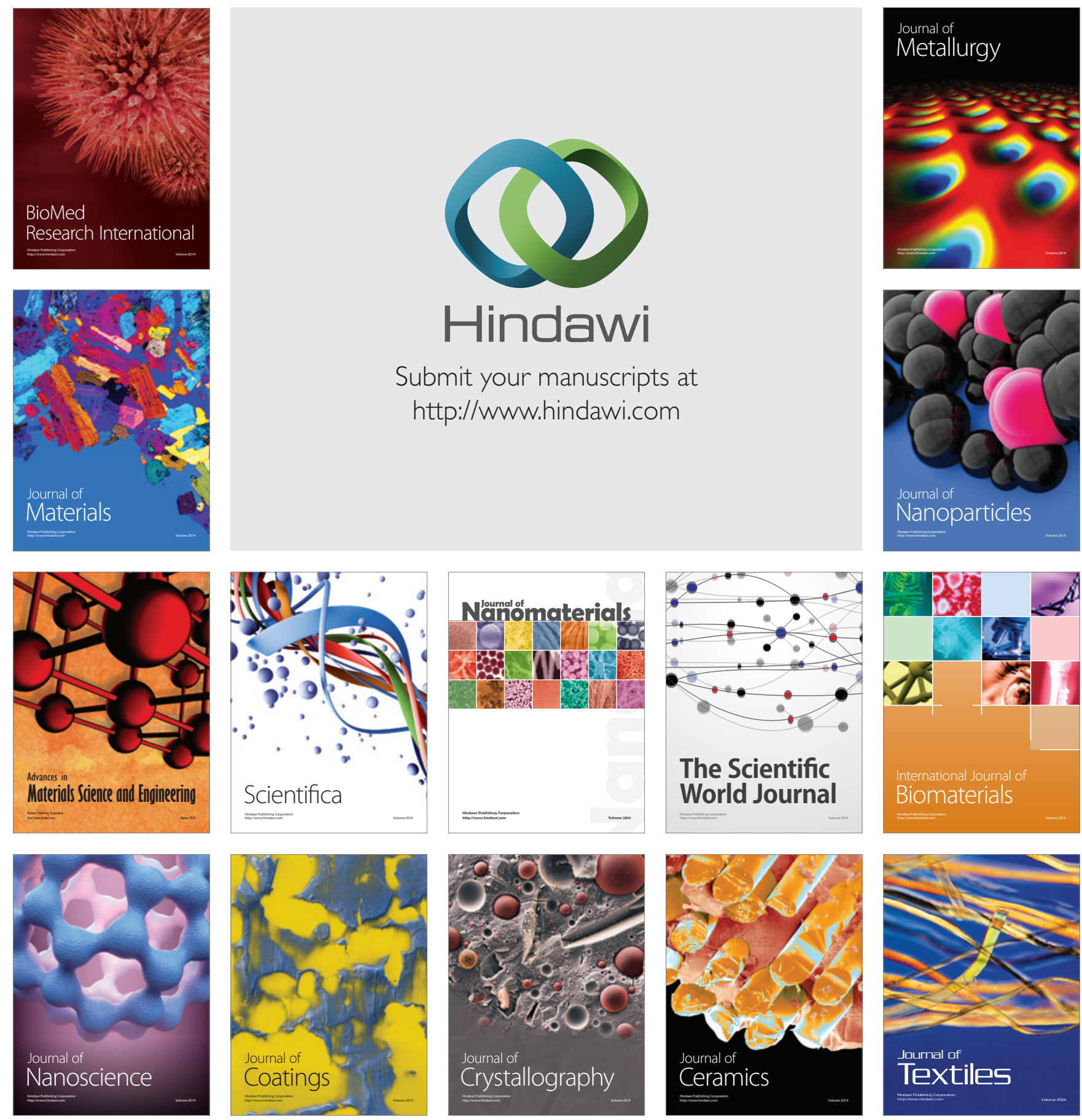